\title{
EDITORIAL
}

\section{Neurosurgical Focus yearly summary}

\author{
Martin H. Weiss, MD, Editor-in-Chief, Neurosurgical Focus \\ Department of Neurosurgery, University of Southern California, Los Angeles, California
}

$\mathrm{T}$ HE remarkable success of Neurosurgical Focus in becoming the world's most widely read electronic neurosurgical journal is dependent upon three distinct constituent elements contributing to each Topic publication. These three elements are the authors of manuscripts submitted for review and potential publication; the editorial review board members (along with select outside reviewers), who provide meticulous reviews of manuscripts submitted for each monthly Topic; and the editorial staff in our Charlottesville office, who carefully review and edit each manuscript accepted for publication, including a process of bibliographic verification that is unique to publications of The Journal of Neurosurgery Publishing Group (JNSPG) amongst all neurosurgical publications.

Each manuscript submitted to Neurosurgical Focus is peer-reviewed by three or four experts before a decision is made to accept, revise, or reject a given submission. Each reviewer, in turn, has a 5-day "turnaround" time to complete an initial review or review of a revision to ensure that we meet our rapid publication deadlines. The competition is keen, requiring detailed analysis of each manuscript to ensure a publication of the highest order.

We are grateful to the following individuals who have devoted their valuable time to ensure the success of $\mathrm{Neu}$ rosurgical Focus during 2014:

Frank Acosta, University of Southern California, Los Angeles, CA

Arun Amar, University of Southern California, Los Angeles, CA

Christopher Ames, University of California, San Francisco, CA

Paul Anderson, University of Wisconsin, Madison, WI

Paul Arnold, University of Kansas Medical Center, Kansas City, KS

Anthony Asher, Carolina Neurosurgery and Spine Associates, Charlotte, $\mathrm{NC}$
Mitchel Berger, University of California, San Francisco, CA

Robert Brown, Mayo Clinic, Rochester, MN

Jeffrey Bruce, Columbia University, New York, NY

Paolo Cappabianca, Università degli Studi di Napoli Federico II, Naples, Italy

Larry Carson, University of North Carolina, Chapel Hill, $\mathrm{NC}$

Thomas Chen, University of Southern California, Los Angeles, $\mathrm{CA}$

Aaron Cohen-Gadol, Goodman Campbell Brain and Spine, Indianapolis, IN

James Cunningham, University of Southern California, Los Angeles, CA

Ralph Dacey, Washington University, St. Louis, MO

Art Day, University of Texas, Houston, TX

Doniel Drazin, Cedars-Sinai Medical Center, Los Angeles, CA

Rick Friedman, University of Southern California, Los Angeles, CA

Steve Giannotta, University of Southern California, Los Angeles, CA

James Goodrich, Montefiore Medical Center, Bronx, NY

Constantinos Hadjipanayis, Emory University School of Medicine, Atlanta, GA

Fred Hagigi, University of California, Los Angeles, CA

Robert Heary, Rutgers New Jersey Medical School, Newark, NJ

Patrick Hsieh, University of Southern California, Los Angeles, CA

Thomas Jackiewicz, University of Southern California Health Care, Los Angeles, CA

John Jane Jr., University of Virginia, Charlottesville, VA

John Jane Sr., University of Virginia, Charlottesville, VA

J. Patrick Johnson, University of California Davis, Sacramento, CA

Adam Kanter, University of Pittsburgh, PA 
Alex Khalessi, University of California, San Diego, CA Terrence Kim, Cedars-Sinai Medical Center, Los Angeles, CA

Giuseppe Lanzino, Mayo Clinic, Rochester, MN

Meng Law, University of Southern California, Los Angeles, CA

Michael Lawton, University of California, San Francisco, CA

Mark Liker, University of Southern California, Los Angeles, CA

John Liu, University of Southern California, Los Angeles, CA

Russell Lonser, Ohio State University, Columbus, Ohio

William Mack, University of Southern California, Los Angeles, CA

Neil Martin, University of California, Los Angeles, CA

Ian McCarthy, Baylor Health Care System, Dallas, TX

Paul McCormick, Columbia University, New York, NY

Nancy McLaughlin, University of California, Los Angeles, CA

Fredric Meyer, Mayo Clinic, Rochester, MN

Praveen Mummaneni, University of California, San Francisco, CA

Edward Oldfield, University of Virginia, Charlottesville, VA
David Polly, University of Minnesota, Minneapolis, MN

Charles Prestigiacomo, Rutgers New Jersey Medical School, Newark, NJ

David Roberts, Dartmouth-Hitchcock Medical Center, Lebanon, $\mathrm{NH}$

Jonathan Russin, University of Southern California, Los Angeles, CA

Theodore Schwartz, Weill Cornell Medical College, New York, NY

Christopher Shaffrey, University of Virginia, Charlottesville, VA

Robert Spetzler, Barrow Neurological Institute, Phoenix, AZ

Robert Starke, University of Virginia, Charlottesville, VA

Victor Tabbush, University of California, Los Angeles, CA

Juan Uribe, University of South Florida, Tampa, FL

Michael Wang, University of Miami, Miami, FL

Gabriel Zada, University of Southern California, Los Angeles, CA

Gelareh Zadeh, University of Toronto, ON, Canada

http://thejns.org/doi/abs/10.3171/2014.10.FOCUS14714 